\title{
Potencial de economia de energia elétrica através do uso da luz natural e da ventilação híbrida em edifícios comerciais em Florianópolis
}

\author{
Electricity savings potential by using daylighting and \\ hybrid ventilation in commercial buildings in \\ Florianópolis
}

\section{Ricardo Forgiarini Rupp Enedir Ghisi}

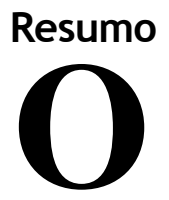
simulados modelos de ambientes de edificações comerciais, com três geometrias, três dimensões de sala por geometria, dez áreas de janela por modelo e quatro orientações. Os modelos foram examinados por meio de quatro estudos de caso. No Caso 1 (referência), a edificação opera com sistemas de iluminação e de condicionamento artificiais; no Caso 2, ocorre a integração da iluminação natural com a artificial, com condicionamento artificial; já no Caso 3, utilizam-se a ventilação híbrida e a iluminação artificial; no Caso 4, adotam-se a iluminação natural integrada com a artificial e a ventilação híbrida. Os consumos de eletricidade do Caso 1 foram comparados com os demais casos. Assim, foi estimado o potencial de economia de energia elétrica gerado pelo uso da luz natural e ventilação híbrida. Conclui-se que a utilização da iluminação natural e da ventilação híbrida em edificações comerciais localizadas em Florianópolis apresenta potencial de economia de energia elétrica de até $64,9 \%$ e que essas estratégias podem ser utilizadas para aumentar a eficiência energética desse tipo de edificação.

Palavras-chave: Luz natural. Ventilação híbrida. Potencial de economia de eletricidade.

\begin{abstract}
The aim of this study is to estimate the electricity savings potential by using daylighting integrated with artificial lighting systems and hybrid ventilation in commercial buildings located in Florianópolis, Brazil. This research work was based on computer simulations using EnergyPlus and Daysim programmes. Commercial building models with three geometries, three room sizes per geometry, four orientations and ten window areas per model were simulated. The models were examined in four case studies. In Case 1 (reference) the building

operates with artificial lighting and air-conditioning systems. Case 2 presents the integration of daylight and artificial light with air-conditioning. In Case 3 hybrid ventilation and artificial lighting were used. In Case 4, the integration of daylight and artificial light with hybrid ventilation was used. The electricity consumption in Case 1 were compared with those in the other cases. Hence, the potential for electricity savings by using daylighting and hybrid ventilation was estimated. The main conclusion was that the use of daylighting and hybrid ventilation in commercial buildings located in Florianópolis presents a potential of up to $64.9 \%$ savings in electricity and such strategies can be used to improve energy efficiency in this type of buildings.

Keywords: Daylighting. Hybrid ventilation. Electricity savings potential.
\end{abstract}

Ricardo Forgiarini Rupp Universidade Federal de Santa Catarina

Florianópolis - SC - Brasil

Enedir Ghisi Universidade Federal de Santa
Catarina
Florianópolis - SC - Brasil Universidade Federal de Santa
Catarina
Florianópolis - SC - Brasil

Recebido em 19/03/13 Aceito em 09/09/13

RUPP, R. F.; GHISI, E. Potencial de economia de energia elétrica através do uso da luz natural e da ventilação híbrida 75 em edifícios comerciais em Florianópolis. Ambiente Construído, Porto Alegre, v. 13, n. 4, p. 75-86, out./dez. 2013. ISSN 1678-8621 Associação Nacional de Tecnologia do Ambiente Construído. 


\section{Introdução}

No Brasil, de acordo com o Balanço Energético Nacional (BALANÇO..., 2012) de 2012, os setores residencial, comercial e público consomem cerca de $14 \%$ do dispêndio final de energia e $47 \%$ do consumo total de eletricidade do país. Com relação aos setores comerciais e públicos, cerca de $70 \%$ dessa energia é destinada aos sistemas de iluminação artificial e ar condicionado, conforme dados de 2007 do Programa Nacional de Conservação de Energia Elétrica (Procel) (PROGRAMA..., 2007), o que vai ao encontro dos dados apresentados em escala mundial (ENERGY..., 2008).

Diante dessa situação surge a necessidade de se estudarem estratégias que visem à economia de energia em iluminação artificial e em sistemas de ar condicionado, os dois grandes consumidores de energia em edificações comerciais. Entre essas estratégias se destacam o uso da iluminação natural integrada à iluminação artificial e da ventilação natural como forma de reduzir a carga térmica da edificação e, consequentemente, o consumo em sistemas de ar condicionado.

Nos últimos anos, vários estudos avaliaram a incorporação da iluminação natural como alternativa para reduzir o consumo de energia em edificações (BODART; HERDE, 2002; KRARTI; ERICKSON; HILLMAN, 2005; ROISIN et al., 2008). O estudo de Souza (2003), em diferentes modelos de salas em Florianópolis, estimou a economia de energia elétrica para 63 modelos de salas que possuíam largura fixa de $6 \mathrm{~m} \mathrm{e}$ profundidades que variavam de $4 \mathrm{~m}$ a $10 \mathrm{~m}$, com três tipos de iluminação e três tipos de janelas. Através dos resultados das simulações nos programas LuzSolar e LuzPALN, constatou-se que a luz natural proporciona uma economia de energia elétrica gasta em iluminação artificial de até $87 \%$.

Já Didoné e Pereira (2010) estudaram, por meio de simulações computacionais nos programas EnergyPlus e Daysim, o impacto do aproveitamento da luz natural no consumo energético de modelos de salas de edificações comerciais localizadas em Florianópolis. Foram estudados quatro modelos de sala, nas quatro orientações principais, com diferentes profundidades, alturas (pé-direito), percentuais de área de abertura na fachada, fatores solares, ângulos de sombreamento horizontais e verticais. Para cada um desses quatro modelos, foi criado um modelo-base, com baixa eficiência energética e sem aproveitamento de luz natural. Os autores concluíram que o controle do sistema de iluminação artificial em função do aproveitamento da luz natural proporcionou uma redução entre
$12 \%$ e $52 \%$ do consumo final de energia elétrica, em comparação ao modelo-base.

Do mesmo modo, sistemas de ventilação natural estão sendo cada vez mais incorporados às edificações comerciais com o objetivo de remover a carga térmica e, consequentemente, reduzir o consumo de energia em sistemas de ar condicionado. Porém, a ventilação natural, promovida por aberturas nas fachadas, tem sua aplicação limitada a alguns climas, locais e tipos de edifícios, por razões de conforto térmico. Assim, com o intuito de ampliar a faixa de aplicação da ventilação natural, a estratégia de ventilação híbrida pode ser utilizada. Nos edifícios híbridos, o sistema natural de ventilação (janelas operáveis) é integrado com o sistema mecânico de ventilação, que é utilizado para resfriar e distribuir melhor o ar (LOMAS; COOK; FIALA, 2007; BRAGER; BORGESON; LEE, 2007).

Diversos autores estudaram o desempenho de sistemas híbridos de ventilação (MANKIBI et al., 2006; BRAGER; BAKER, 2008; NIACHOU et al., 2008). Karava et al. (2012) estudaram um sistema híbrido de ventilação em um edifício comercial em Montreal, Canadá. A ventilação foi promovida mecanicamente por meio de aberturas (em forma de grelhas) no piso, nas fachadas e no átrio. O sistema híbrido de ventilação operou automaticamente em função das condições climáticas externas. Quando a temperatura externa estava entre 15 e $25^{\circ} \mathrm{C}$ e a umidade relativa era menor que $70 \%$, as grelhas eram abertas, caso contrário permaneciam fechadas. Para avaliar o desempenho do sistema híbrido, sensores para a medição de diferentes variáveis climáticas internas e externas foram utilizados. Baseando-se nos dados medidos, os autores calcularam o potencial de economia em resfriamento devido ao uso do sistema híbrido, que foi de $30 \%$, quando comparado ao sistema mecânico de resfriamento.

Outro estudo sobre ventilação híbrida foi realizado por Ji, Lomas e Cook (2009) em um edifício de baixo consumo de energia em Hangzhou (sul da China). O sistema mecânico operava durante o verão e o inverno, e, nos demais períodos do ano, era utilizado o sistema de ventilação natural. O sistema de ventilação natural foi modelado por dinâmica dos fluidos computacional pelo programa ANSYS CFX, e o desempenho térmico do edifício, pelo programa IES Virtual Environment. Os resultados de potenciais de economia em resfriamento do sistema híbrido foram da ordem de 30-35\%, quando comparados ao sistema mecânico operando o ano inteiro. Os autores concluíram ainda que o sistema híbrido de ventilação se

76 Rupp, R. F.; Ghisi, E. 
mostrou viável, mesmo no clima subtropical úmido do sul da China.

Os trabalhos mencionados demonstraram que há potencial de economia de energia elétrica através do aproveitamento da luz natural ou por meio do uso de ventilação híbrida. Do mesmo modo, este trabalho tem como intuito colaborar para esse campo de pesquisa, estimando o potencial de economia quando se faz uso do aproveitamento da luz natural e da ventilação híbrida simultaneamente.

O objetivo deste artigo é estimar o potencial de economia de energia elétrica com o uso da luz natural integrada ao sistema de iluminação artificial e a utilização da ventilação híbrida em edifícios comerciais localizados em Florianópolis, SC.

\section{Método}

A pesquisa é de caráter quantitativo e é baseada em resultados de simulações computacionais de modelos de edificações comerciais localizadas em Florianópolis, nos programas EnergyPlus 6.0 (ENERGY..., 2010) e Daysim 3.0 (DAYSIM, 2011). O arquivo climático de Florianópolis TRY 1963 (LABORATÓRIO..., 2011a) foi utilizado para as simulações, considerando as 8.760 horas do ano.

\section{Modelos de estudo}

Foram estudados diferentes modelos de ambientes de edifícios comerciais, conforme a Tabela 1. Cada caso simulado corresponde ao desempenho de uma única sala, na qual o teto, o piso e as paredes internas são adiabáticos. Com o intuito de avaliar a influência que diferentes geometrias exercem no potencial de economia de energia elétrica, foram definidas três geometrias nas proporções (largura:profundidade) de 2:1, 1:1 e 1:2. As geometrias foram fundamentadas no índice de ambiente (K), descrita em manuais luminotécnicos (Equação 1). Nestes, os índices de ambiente variam entre 0,6 e 5,0. Neste trabalho, são estudados três índices: 0,8, 2,0 e 5,0. A altura dos ambientes foi fixada em $2,80 \mathrm{~m}$, e a altura do plano de trabalho, em $0,75 \mathrm{~m}$ em relação ao piso. Em cada modelo (Tabela 1) foram variadas as áreas de janela de $10 \%$ a $100 \%$ da área útil de janela, com intervalos de $10 \%$, e a orientação da fachada envidraçada (norte, sul, leste e oeste), conforme a Figura 1. A janela começa a $60 \mathrm{~cm}$ da parte superior da fachada e possui a largura da fachada. A área útil de janela corresponde ao total de área da fachada, que pode ser envidraçada, ou seja, desconta-se da área total da fachada a parcela relativa à viga.

$\mathrm{K}=\frac{\mathrm{L} \cdot \mathrm{P}}{(\mathrm{L}+\mathrm{P}) \cdot \mathrm{h}}$

Eq. 1

Onde:

$L$ é a largura da sala (metros);

$P$ é a profundidade da sala (metros); e

$h$ é a altura de montagem entre a superfície de trabalho e o teto (metros).

Tabela 1 - Dimensões dos modelos para cada índice de ambiente e geometria

\begin{tabular}{c|c|c|c|c|c|c}
\hline \multirow{2}{*}{$\mathbf{K}$} & \multicolumn{6}{|c}{ Geometria - Largura (L):Profundidade (P) } \\
\cline { 2 - 7 } & \multicolumn{2}{|c|}{$\mathbf{2}$} & \multicolumn{2}{c}{$\mathbf{1 : 1}$} & \multicolumn{2}{|c}{$\mathbf{1 : 2}$} \\
\cline { 2 - 7 } & $\mathbf{L}(\mathbf{m})$ & $\mathbf{P}(\mathbf{m})$ & $\mathbf{L}(\mathbf{m})$ & $\mathbf{P}(\mathbf{m})$ & $\mathbf{L}(\mathbf{m})$ & $\mathbf{P}(\mathbf{m})$ \\
\hline 0,8 & 4,92 & 2,46 & 3,28 & 3,28 & 2,46 & 4,92 \\
2,0 & 12,30 & 6,15 & 8,20 & 8,20 & 6,15 & 12,30 \\
5,0 & 30,75 & 15,38 & 20,50 & 20,50 & 15,38 & 30,75 \\
\hline
\end{tabular}

Figura 1 - Áreas úteis de janela analisadas

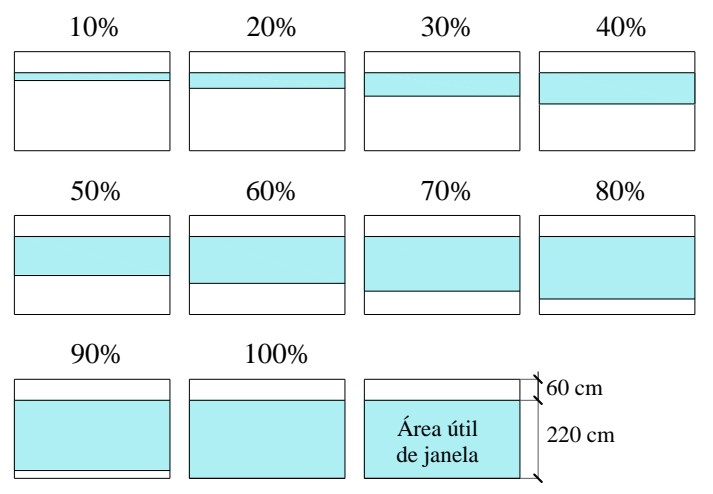


Cada modelo de estudo (Tabela 1) foi investigado por meio de quatro estudos de caso:

(a) caso 1 (referência): o modelo opera com sistemas de iluminação e de condicionamento artificiais;

(b) caso 2: no modelo ocorre a integração da iluminação natural com a artificial, com condicionamento artificial;

(c) caso 3: o modelo utiliza ventilação híbrida e iluminação artificial; e

(d) caso 4: o modelo utiliza a iluminação natural integrada com a artificial e a ventilação híbrida.

\section{Simulações computacionais}

A seguir são apresentadas as considerações que foram adotadas para as simulações dos quatro estudos de caso nos programas EnergyPlus e Daysim, para as três geometrias, três dimensões de sala por geometria, dez áreas de janela por ambiente e quatro orientações. Informações mais detalhadas das simulações podem ser consultadas em Rupp (2011).

\section{Parâmetros gerais de simulação}

Em todos os modelos se consideraram iluminação artificial e cargas internas (Tabela 2) funcionando das $8 \mathrm{~h}$ às $18 \mathrm{~h}$ de segunda a sexta-feira (períodos de ocupação do edifício). As densidades de potência em iluminação (Tabela 3) foram determinadas para cada ambiente a partir de projeto luminotécnico, que foi realizado pelo método dos lumens, de modo a garantir um nível de iluminância mínimo de 500 lux na superfície de trabalho. Foram utilizadas lâmpadas fluorescentes tubulares TL528W-HE/840 e luminárias modulares de embutir EcoFIX TBS262.

As características dos componentes construtivos (Tabela 4) foram baseadas no trabalho de Santana (2006), com exceção do vidro (vidro simples, 6 $\mathrm{mm}, 88 \%$ de transmissão luminosa), que foi fundamentado na base de dados do EnergyPlus (2010).

Tabela 2 - Cargas internas dos ambientes

\begin{tabular}{l|c|c}
\hline \multicolumn{1}{c|}{ Cargas internas } & Valor & Fonte \\
\hline Ocupação $\left(\mathrm{m}^{2} /\right.$ pessoa) & 14,7 & Santana $(2006)$ \\
Atividade $\left(\mathrm{W} / \mathrm{m}^{2}\right)$ & 65,0 & Standard 55 (AMERICAN..., 2010) \\
Equipamentos $\left(\mathrm{W} / \mathrm{m}^{2}\right)$ & 9,7 & Santana $(2006)$ \\
\hline
\end{tabular}

Tabela 3 - Densidades de potência em iluminação, DPI (W/m²)

\begin{tabular}{c|c|c|c}
\hline \multirow{2}{*}{ K } & \multicolumn{3}{|c}{ Geometria - Largura (L):Profundidade (P) } \\
\cline { 2 - 4 } & $\mathbf{2 : 1}$ & $\mathbf{1 : 1}$ & $\mathbf{1 : 2}$ \\
\hline 0,8 & 13,9 & 15,6 & 13,9 \\
2,0 & 9,6 & 9,2 & 9,6 \\
5,0 & 8,1 & 8,0 & 8,1 \\
\hline
\end{tabular}

Tabela 4 - Propriedades dos componentes construtivos

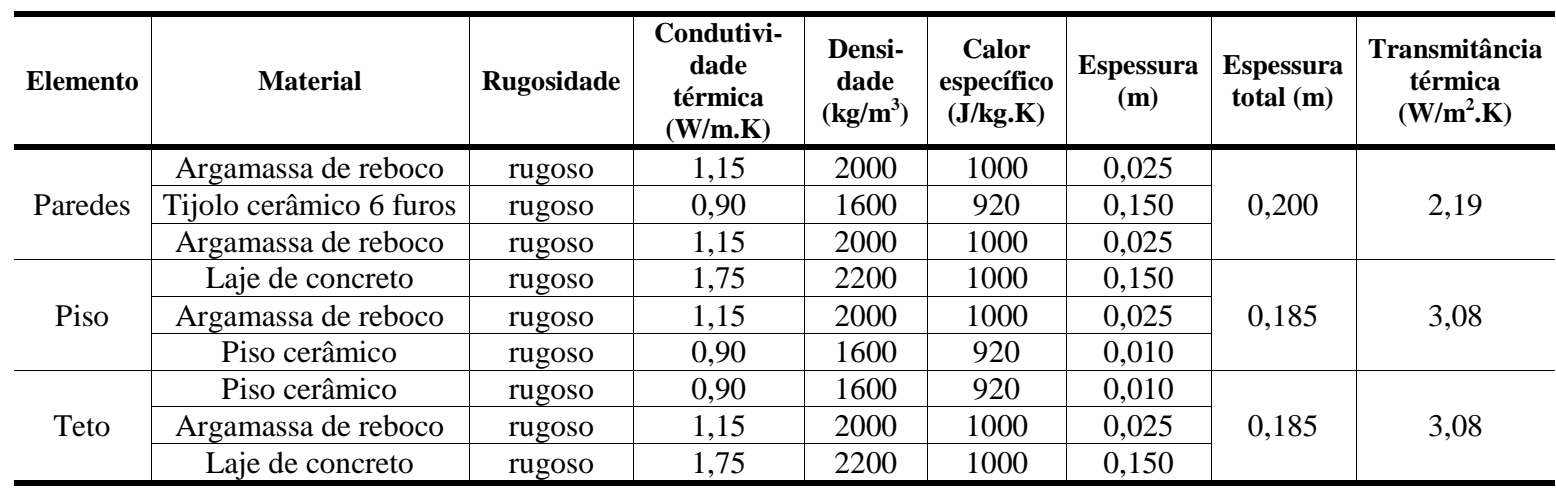

Fonte: baseado em Santana (2006). 
O sistema de ar condicionado consiste em um aparelho do tipo split funcionando com compressor externo e foi modelado no EnergyPlus através do template HVACTemplateZone:PTHP. Consideraram-se um COP (Coefficient Of Performance) de 3,28 e uma taxa de renovação de ar interior de $0,0075 \mathrm{~m}^{3} / \mathrm{s} /$ pessoa. A temperatura de setpoint do sistema de ar condicionado foi definida em $24^{\circ} \mathrm{C}$ durante os períodos de ocupação do edifício ( $8 \mathrm{~h}$ às $18 \mathrm{~h}$ de segunda a sexta-feira). $\mathrm{O}$ sistema de ar condicionado foi utilizado somente na função de resfriamento, porque a função de aquecimento não é usada em edifícios comerciais em Florianópolis, de acordo com Santana (2006).

\section{Simulações com luz natural}

As simulações de iluminação natural (Casos 2 e 4) foram realizadas no Daysim. Este programa foi utilizado para simular a iluminação natural, porque alguns autores demonstraram que o algoritmo de luz natural do EnergyPlus superestima as iluminâncias internas (LOUTZENHISER; MAXWELL; MANZ, 2007; RAMOS; GHISI, 2010).

Nos modelos, os sensores de luz natural foram mantidos distanciados entre si em $0,2 \mathrm{~m}$, criando uma malha de pontos equidistantes. As refletâncias internas foram definidas em $80 \%$ (teto), $50 \%$ (paredes) e $30 \%$ (piso). O controle da iluminação artificial foi feito através de um sistema dimerizável, baseando-se nos valores de iluminação natural, tendo sido o nível de iluminância mínimo a ser garantido de 500 lux, conforme recomendações da NBR ISO/CIE 89951 (ABNT, 2013) para atividades de escritório.

A integração da iluminação natural e artificial foi feita por meio da rotina de controle da iluminação artificial proveniente das simulações no Daysim, de cada modelo dos casos com luz natural. O Daysim gera automaticamente uma rotina em base horária para as $8.760 \mathrm{~h}$ do ano (em um arquivo no formato $\mathrm{CSV}$ ), de funcionamento da iluminação artificial, com valores entre 0 (o nível de iluminância requerido é totalmente satisfeito pela iluminação natural, ou seja, a iluminação artificial é desligada) e 1 (a iluminação artificial é ligada na potência máxima). Os valores intermediários utilizam a integração entre a iluminação natural e a artificial, sendo a potência desta última regulada de maneira a garantir o nível de iluminância mínimo de 500 lux. Essa rotina foi inserida como dado de entrada no EnergyPlus, através do componente Schedule: File, sendo esta selecionada em Schedule Name dentro do parâmetro Lights. Dessa forma, a potência em iluminação artificial foi controlada por esta rotina para cada modelo dos casos com luz natural.

Não foram considerados elementos de proteção à iluminação excessiva nos espaços. $\mathrm{Na}$ prática, o uso de tais elementos reduzirá o aproveitamento de luz natural.

\section{Simulações com ventilação híbrida}

A simulação da ventilação natural foi realizada por meio do modelo Airflow Network, multizona, e os coeficientes de pressão do vento foram calculados pelo próprio EnergyPlus. A ventilação natural foi incorporada sempre que foram atendidos os requisitos de abertura de janela coincidentes com as rotinas calculadas de ventilação. Os modelos de ambientes de edifícios comerciais, por suas características, não permitem a ventilação cruzada, o que pode reduzir significativamente o aproveitamento da ventilação natural. Assim, os consumos de eletricidade em sistema de ar condicionado seriam afetados nos Casos 3 e 4.

Com o intuito de incorporar a ventilação híbrida, rotinas de funcionamento do sistema de ar condicionado e rotinas de funcionamento da ventilação natural foram criadas, em base horária para as $8.760 \mathrm{~h}$ do ano, em planilhas, e utilizadas como dados de entrada no EnergyPlus. A rotina de funcionamento da ventilação natural é o inverso da rotina de funcionamento do sistema de ar condicionado, ou seja, quando se permite a ventilação natural, restringe-se o uso do sistema de ar condicionado, e vice-versa. Os procedimentos de "a" a "c" foram utilizados para a determinação dessas rotinas de funcionamento durante os períodos de ocupação do edifício:

(a) simulação da ventilação natural: as janelas foram consideradas operáveis e foram abertas, e o sistema de ar condicionado, desligado, quando três requisitos foram cumpridos: (1) a temperatura da zona foi maior que a temperatura externa; (2) a temperatura da zona foi maior que a temperatura de setpoint para a ventilação natural; e (3) a rotina de controle da ventilação natural permitiu a abertura de janelas. As temperaturas de setpoint para a ventilação natural foram de $22^{\circ} \mathrm{C}$ (período de inverno, de $21 / 03$ a 20/09) e de $20^{\circ} \mathrm{C}$ (período de verão, de 21/09 a 20/03), de acordo com recomendações de Sorgato (2009). Os coeficientes e expoentes do fluxo de ar, assim como os coeficientes de descarga são dados pela Tabela 5. As rotinas de funcionamento da ventilação natural permitiram a ventilação das $8 \mathrm{~h}$ às $18 \mathrm{~h}$, de segunda a sexta-feira. Para cada modelo, a temperatura de bulbo seco (TBS), a umidade relativa do ar (UR) e a umidade absoluta (UA) foram obtidas em base horária. 
(b) Comparação de TBS, UR e UA com o método de avaliação de conforto térmico: os valores de temperatura de bulbo seco, de umidade absoluta e de umidade relativa obtidos das simulações de ventilação natural foram comparados com os limites superiores da zona de conforto térmico da carta de Givoni (1992). O método de Givoni foi escolhido por dois motivos principais: (1) atualmente não existe um método apropriado para ser utilizado em edifícios comerciais com ventilação híbrida; e (2) baseando-se em estudos que apontaram que o método de conforto adaptativo da ASHRAE 55 (AMERICAN..., 2010) para ambientes ventilados naturalmente não estipula limites máximos de umidade, pode apresentar problemas de umidade (condensação, degradação de materiais e contaminação biológica) em climas úmidos (EMMERICH; POLIDORO; AXLEY, 2011). Assim, um limite de umidade deveria ser utilizado (EMMERICH; POLIDORO; AXLEY, 2011). Rupp e Ghisi (2014) avaliaram o conforto térmico de diferentes modelos de edifícios comerciais com ventilação híbrida em Florianópolis pelo método da ASHRAE 55 (AMERICAN..., 2010) para ambientes ventilados naturalmente e pelo método de Givoni (1992) adaptado para climas quentes e úmidos. Os autores (RUPP; GHISI, 2014) concluíram que o método da ASHRAE 55 (AMERICAN..., 2010) apresentou pouca ocorrência de desconforto térmico por calor no verão de Florianópolis (o que resultaria em poucas horas de uso do ar condicionado), enquanto o método de Givoni se mostrou mais adequado, por ter um limite de umidade estipulado. Assim, o limite máximo aceitável de UR foi de $80 \%$, e até os $27^{\circ} \mathrm{C}$ de TBS o limite máximo aceitável de UA foi de $17 \mathrm{~g} / \mathrm{kg}$. De $27^{\circ} \mathrm{C}$ até $29^{\circ} \mathrm{C}$ de TBS, os limites máximos aceitáveis de UA são dados pela Equação 2, obtida através da carta de Givoni (1992):

$\mathrm{UA}=-2,25 . \mathrm{TBS}+77,75,27^{\circ} \mathrm{C}<\mathrm{TBS}<29^{\circ} \mathrm{C}$ Eq. 2
Onde:

$U A$ é a umidade absoluta $(\mathrm{g} / \mathrm{kg})$; e

TBS é a temperatura de bulbo seco $\left({ }^{\circ} \mathrm{C}\right)$.

(c) permissão da ventilação natural ou do sistema de ar condicionado: o sistema de ar condicionado foi ligado quando os valores de TBS, UA e UR foram maiores que os limites máximos aceitáveis para conforto. Quando esses valores foram menores que os limites máximos aceitáveis, a ventilação natural foi permitida.

A ventilação natural também foi permitida nos demais horários de cada dia da semana, quando se satisfizeram os três requisitos descritos em "a". Nos fins de semana, tanto o sistema de ar condicionado quanto a ventilação natural não foram acionados.

\section{Parâmetros de análise}

Para cada caso simulado foi solicitado um relatório com o consumo total anual de eletricidade $\left(\mathrm{kWh} / \mathrm{m}^{2}\right)$, englobando o consumo em iluminação artificial, em equipamentos e em sistema de ar condicionado. Com base nesses resultados, foram feitas comparações de consumo de energia elétrica entre o Caso 1 e os demais casos. Essa comparação foi feita para determinar o potencial de economia de energia elétrica e foi baseada na redução no consumo de eletricidade, calculada através da Equação 3.

$\mathrm{RC}=\left(1-\frac{\mathrm{CC}}{\mathrm{C} 1}\right) \times 100$

Onde:

$R C$ é a redução no consumo de energia entre os estudos de caso (\%);

Cl é o consumo de eletricidade do Caso 1 $\left(\mathrm{kWh} / \mathrm{m}^{2}\right)$; e

$C C$ é o consumo de eletricidade para o caso a ser comparado com o Caso $1\left(\mathrm{kWh} / \mathrm{m}^{2}\right)$.

Tabela 5 - Parâmetros da janela para a simulação da ventilação natural no EnergyPlus

\begin{tabular}{c|c}
\hline Parâmetro & Característica \\
\hline Descrição do componente & $\begin{array}{c}\text { Janela de metal, 1 } \\
\text { folha, deslizamento } \\
\text { horizontal }\end{array}$ \\
\hline Coeficiente do fluxo de ar quando a abertura está fechada $(\mathrm{kg} / \mathrm{s} . \mathrm{m})$ & 0,00010 \\
Expoente do fluxo de ar quando a abertura está fechada (adimensional) & 0,66 \\
Número de frações de abertura & 2 \\
Fator de abertura 1 - Janela fechada (adimensional) & 0 \\
Coeficiente de descarga para o fator de abertura 1 (adimensional) & 0,001 \\
Fator de abertura 2 - Janela aberta (adimensional) & 1 \\
Coeficiente de descarga para o fator de abertura 2 (adimensional) & 0,6 \\
\hline
\end{tabular}

Fonte: baseado em Liddament (1986).

80 Rupp, R. F.; Ghisi, E. 
Para o período de ocupação do edifício foram obtidas, através das simulações, para cada modelo de cada um dos quatro estudos de caso, temperatura de bulbo seco $\left({ }^{\circ} \mathrm{C}\right)$, umidade relativa do ar $(\%)$ e umidade absoluta do ar $(\mathrm{g} / \mathrm{kg})$, para o ambiente interno. Esses valores foram introduzidos como dado de entrada no programa Analysis Bio 2.2 (LABORATÓRIO..., 2011b) que plota essas variáveis sob a carta de Givoni (1992), adaptada para países de clima quente e úmido. Assim, foi possível analisar, comparando com o Caso 1, as variações de temperatura e umidade quando se utilizam a iluminação natural e a ventilação híbrida, e quando as duas estratégias são usadas simultaneamente.

\section{Resultados}

A seguir são mostrados os consumos de energia elétrica para cada estudo de caso. A partir dos consumos de eletricidade foi possível obter os potenciais de economia de energia elétrica com relação ao Caso 1 (referência), os quais são apresentados nesta parte do trabalho.

Os resultados de consumos máximos e mínimos de energia elétrica obtidos para cada estudo de caso são indicados na Tabela 6. Na Figura 2 apresentase o consumo de energia elétrica, separado por uso final, por área de janela para ambiente com geometria de $2: 1$, orientação sul e índice de ambiente igual a 0,8 . Os máximos potenciais de economia de energia elétrica com relação ao Caso 1 são dados pela Tabela 7 .

Para o Caso 1, como os consumos em iluminação e equipamentos são constantes, ao aumentar a área de janela, aumenta-se o consumo de eletricidade do sistema de ar condicionado, com comportamento linear (Figura 2). Assim, quanto maior a área de janela, maior o consumo de energia elétrica (com índice de ambiente igual a 5, o aumento do consumo de energia elétrica devido ao aumento da área de janela foi pequeno). Essa elevação no consumo é devida ao aumento da carga térmica interna do ambiente, proveniente do ganho térmico pela janela. Dessa maneira, necessita-se de maior carga de resfriamento, que resulta em um maior consumo do sistema de ar condicionado.

Para o Caso 2, os consumos em iluminação artificial diminuem com o aumento da área de janela, devido ao aproveitamento da luz natural (Figura 2) - os consumos de energia elétrica por área de janela foram muito similares para os modelos com índice de ambiente igual a 5. Com a diminuição do consumo em iluminação artificial, diminui-se a carga térmica interna do ambiente, o que acarreta em uma diminuição do consumo de energia elétrica do sistema de ar condicionado, com relação ao Caso 1 (Figura 2). Mesmo assim, os consumos em sistema de ar condicionado aumentaram gradativamente com o aumento da área de janela (Figura 2). Já com relação ao consumo total de energia elétrica, pode-se chegar a uma redução de até $50,5 \%$, quando se utiliza a integração da luz natural com a iluminação artificial (Tabela 7). As maiores reduções no consumo de energia elétrica ocorreram para a orientação sul (Tabela 7), e as menores, para a orientação oeste. Entretanto, as economias absolutas $\left(\mathrm{kWh} / \mathrm{m}^{2}\right)$ para a orientação sul foram as menores entre as quatro orientações. As reduções entre os índices de ambiente de uma mesma geometria variaram consideravelmente. A geometria exerceu significativa influência na redução do consumo de energia elétrica. Salas menores (com índice de ambiente igual a 0,8) e menos profundas (geometria de 2:1 e 1:1) apresentaram maiores reduções no consumo (maior aproveitamento da luz natural). Os modelos com geometria de 1:2 e índice de ambiente igual a 5 não apresentaram reduções no consumo de eletricidade, em relação ao Caso 1 (referência).

Para o Caso 3, percebe-se que, de modo geral, ocorre um aumento do consumo de energia elétrica com o aumento da área de janela (Figura 2) - para ambientes com índice de ambiente igual a 5, os consumos de energia elétrica por área de janela são muito semelhantes a partir de certa área de janela. Entretanto, com valores de consumo inferiores aos do Caso 1 (Figura 2), chega-se a valores de redução de consumo total de energia elétrica de até $31,9 \%$ (Tabela 7). Através da ventilação natural, diminui-se a carga térmica interna do ambiente. Com a incorporação da ventilação híbrida, alternando entre a ventilação natural e o uso do aparelho de ar condicionado, diminui-se, com relação ao Caso 1, o número de horas que o sistema de ar condicionado estaria ligado. De modo geral, com áreas de janelas superiores, maiores economias de energia elétrica foram obtidas. Ressalta-se que as economias absolutas $\left(\mathrm{kWh} / \mathrm{m}^{2}\right)$ também foram maiores para áreas de janelas superiores. As maiores reduções no consumo de energia elétrica ocorreram para a orientação norte (Tabela 7), e as menores, para a orientação sul. Salas menores (com índice de ambiente igual a 0,8 ) apresentaram as maiores reduções no consumo. Porém, as reduções entre os índices de ambiente de uma mesma geometria variaram pouco, sendo mais significativas para a orientação sul. A geometria exerceu pouca influência na redução no consumo de energia elétrica. 
No Caso 4, o comportamento do consumo em iluminação artificial é similar ao do Caso 2 (Figura 2). Porém, nesse caso, a carga térmica interna do ambiente também é reduzida devido ao uso da ventilação natural. Desse modo, o consumo do sistema de ar condicionado é duplamente afetado, conduzindo aos menores consumos de eletricidade entre os casos estudados (Tabela 4 e Figura 2). Contrariamente aos demais casos, no Caso 4, há uma maior variação dos consumos de energia elétrica por área de janela para os ambientes com índice de ambiente igual a 5. Neste caso, com áreas de janelas superiores obtiveram-se maiores economias de energia elétrica tanto em porcentagem quanto em $\mathrm{kWh} / \mathrm{m}^{2}$. As maiores reduções no consumo de energia elétrica ocorreram para a orientação norte (Tabela 7), e as menores, para a orientação sul. As maiores reduções no consumo foram obtidas para salas menores (com índice de ambiente igual a 0,8). Já com relação às reduções entre os índices de ambiente de uma mesma geometria, o comportamento foi similar ao apresentado para o Caso 2. A geometria influenciou na redução no consumo de energia elétrica; notadamente em ambientes com geometria de 1:2, as economias foram menores em comparação com as geometrias de 2:1 e 1:1. Já o consumo total de energia elétrica pode chegar a uma redução de até $64,9 \%$ (Tabela 7).

Tabela 6 - Consumos máximos e mínimos de energia elétrica para cada estudo de caso

\begin{tabular}{c|c|c|c|c}
\hline Consumo $\left(\mathrm{kWh} / \mathrm{m}^{2}\right.$.ano) & Caso 1 & Caso 2 & Caso 3 & Caso 4 \\
\hline Máximo & 120,47 & 79,62 & 87,36 & 62,73 \\
Mínimo & 61,53 & 39,73 & 51,39 & 34,02 \\
\hline
\end{tabular}

Figura 2 - Consumo de energia elétrica para cada estudo de caso, separado por uso final, por área de janela para ambiente com geometria de $2: 1$, orientação sul e índice de ambiente igual a 0,8

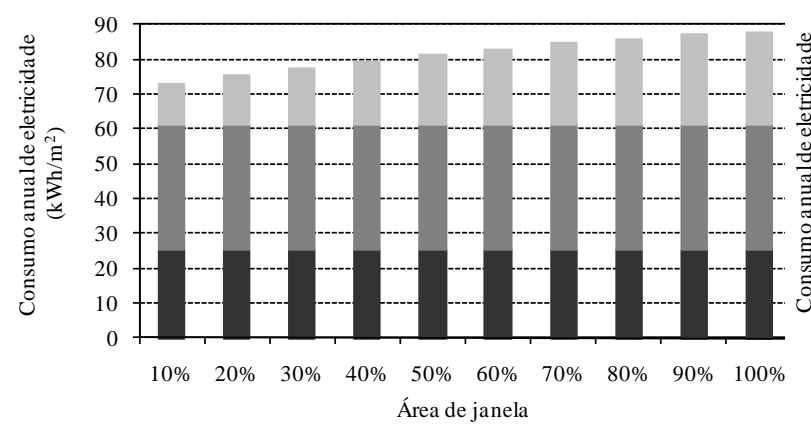

Caso 1

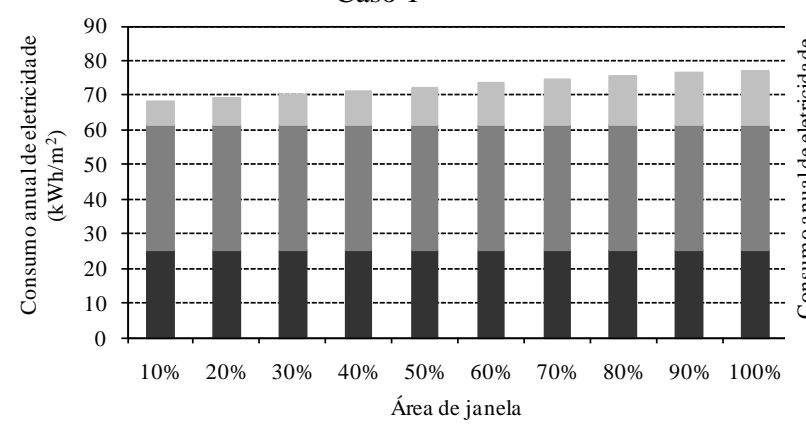

Caso 3

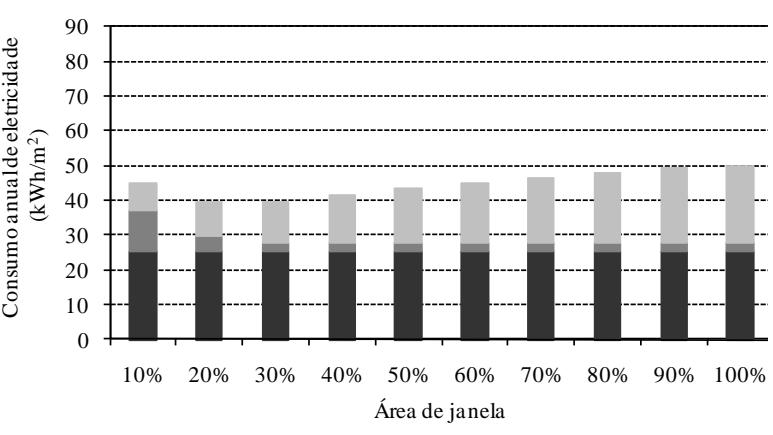

Caso 2

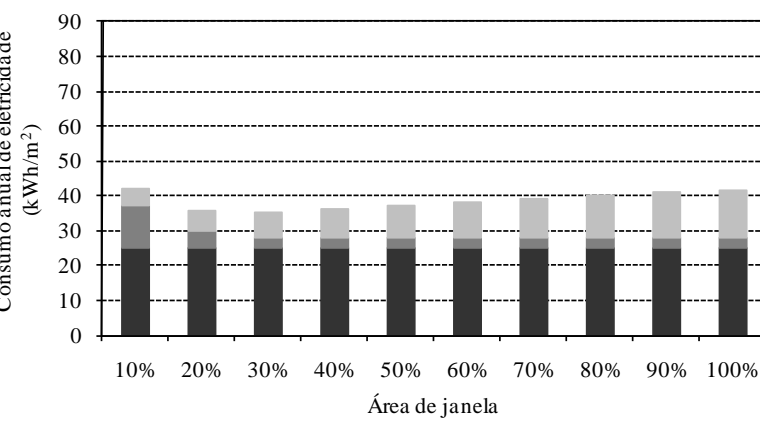

Caso 4

Equipamentos $\square$ lluminação artificial $\square$ Ar-condicionado 
Tabela 7 - Máximo potencial de economia de eletricidade com relação ao Caso 1

\begin{tabular}{|c|c|c|c|c|c|c|c|c|c|c|}
\hline \multirow{3}{*}{ Orientação } & \multirow{3}{*}{ Caso } & \multicolumn{9}{|c|}{ Máximo potencial de economia de eletricidade com relação ao Caso 1 (\%) } \\
\hline & & \multicolumn{3}{|c|}{ Geometria de 2:1 } & \multicolumn{3}{|c|}{ Geometria de 1:1 } & \multicolumn{3}{|c|}{ Geometria de 1:2 } \\
\hline & & $\mathrm{K}=\mathbf{0 , 8}$ & $\mathbf{K}=\mathbf{2 , 0}$ & $K=5,0$ & $\mathrm{~K}=\mathbf{0 , 8}$ & $K=\mathbf{2 , 0}$ & $K=5,0$ & $\mathrm{~K}=\mathbf{0 , 8}$ & $K=\mathbf{2 , 0}$ & $K=5,0$ \\
\hline \multirow{3}{*}{ norte } & 2 & 46,2 & 34,1 & 11,2 & 48,6 & 30,1 & 4,9 & 40,3 & 14,2 & 0,0 \\
\hline & 3 & 31,9 & 28,4 & 26,0 & 30,0 & 28,2 & 25,3 & 29,5 & 26,7 & 24,4 \\
\hline & 4 & 63,3 & 57,1 & 36,0 & 64,9 & 54,7 & 29,6 & 63,3 & 39,5 & 24,4 \\
\hline \multirow{3}{*}{ sul } & 2 & 48,7 & 37,6 & 10,9 & 50,5 & 33,0 & 4,6 & 49,5 & 30,0 & 0,0 \\
\hline & 3 & 12,2 & 14,7 & 18,0 & 13,0 & 16,3 & 18,6 & 14,9 & 17,7 & 19,5 \\
\hline & 4 & 54,8 & 48,6 & 28,0 & 57,9 & 45,8 & 22,8 & 57,3 & 44,8 & 21,7 \\
\hline \multirow{3}{*}{ leste } & 2 & 45,8 & 34,5 & 13,0 & 47,1 & 29,8 & 7,1 & 39,4 & 16,3 & 0,0 \\
\hline & 3 & 26,2 & 24,1 & 23,2 & 25,5 & 24,8 & 23,0 & 25,9 & 23,5 & 22,3 \\
\hline & 4 & 57,4 & 52,8 & 34,7 & 60,5 & 51,2 & 29,1 & 58,5 & 37,5 & 22,3 \\
\hline \multirow{3}{*}{ oeste } & 2 & 45,5 & 34,5 & 12,2 & 46,5 & 29,1 & 4,7 & 38,7 & 13,9 & 0,0 \\
\hline & 3 & 27,6 & 26,8 & 24,7 & 27,5 & 26,9 & 24,3 & 28,2 & 25,9 & 23,5 \\
\hline & 4 & 58,1 & 54,5 & 35,5 & 61,0 & 52,5 & 28,5 & 60,4 & 37,9 & 23,5 \\
\hline
\end{tabular}

Ressalta-se que os potenciais de economia estimados para os casos com iluminação natural (Casos 2 e 4) não consideraram proteção à radiação solar direta nos espaços. $\mathrm{Na}$ prática, para evitar manchas de sol em superfícies de trabalho (probabilidade de ofuscamento), o uso de proteções externas ou internas se faz necessário. Desse modo, os potenciais de economia de eletricidade através do uso de luz natural podem ser menores do que os apresentados neste trabalho.

Os resultados das simulações de cada estudo de caso (TBS, UA e UR) para o período de ocupação do edifício foram plotados sobre a carta de Givoni através do Analysis Bio. Na Figura 3 encontram-se os resultados dos estudos de caso para o ambiente com geometria de 2:1, índice de ambiente igual a 0,8 , orientação oeste e $50 \%$ de área de janela. Nota-se que os Casos 1 e 2 apresentam comportamentos similares; do mesmo modo, os Casos 3 e 4 possuem comportamentos semelhantes. A distribuição dos pontos na carta de Givoni relativa ao Caso 1 e ao Caso 2 apresenta maior controle da umidade. Essa uniformidade é maior para o ambiente maior (índice de ambiente igual a 5). Isso é garantido pelo uso do sistema de ar condicionado durante todo o período de ocupação da edificação. Nos Casos 3 e 4, há maior dispersão dos pontos dentro e fora da zona de conforto térmico, sendo esta mais acentuada nos ambientes com maiores áreas de janela. Essa maior dispersão foi causada pelo uso da ventilação híbrida (alternância entre o acionamento do sistema de ar condicionado e a ventilação natural). Para os demais ambientes e orientações, o comportamento da distribuição dos pontos na zona de conforto foi similar ao apresentado anteriormente.

Para os quatro estudos de caso foi garantida uma elevada porcentagem (acima de 98\%) de número de horas de conforto térmico para os ocupantes durante o período de ocupação do edifício. Nos horários de início de operação do sistema de ar condicionado, em algumas situações, ocorreu desconforto térmico por calor, pois é a partir do acionamento do sistema de ar condicionado que se inicia o processo de resfriamento do ambiente. Em alguns períodos no inverno, mesmo com a janela fechada, o ambiente interno ficou ligeiramente frio, causando leve desconforto térmico por frio. 
Figura 3 - Carta de Givoni (Zona 1 é a zona de conforto térmico) com os resultados das simulações dos quatro estudos de caso, para o período de ocupação do edifício, para o ambiente com geometria de $2: 1$, índice de ambiente igual a 0,8 , orientação oeste e $50 \%$ de área de janela

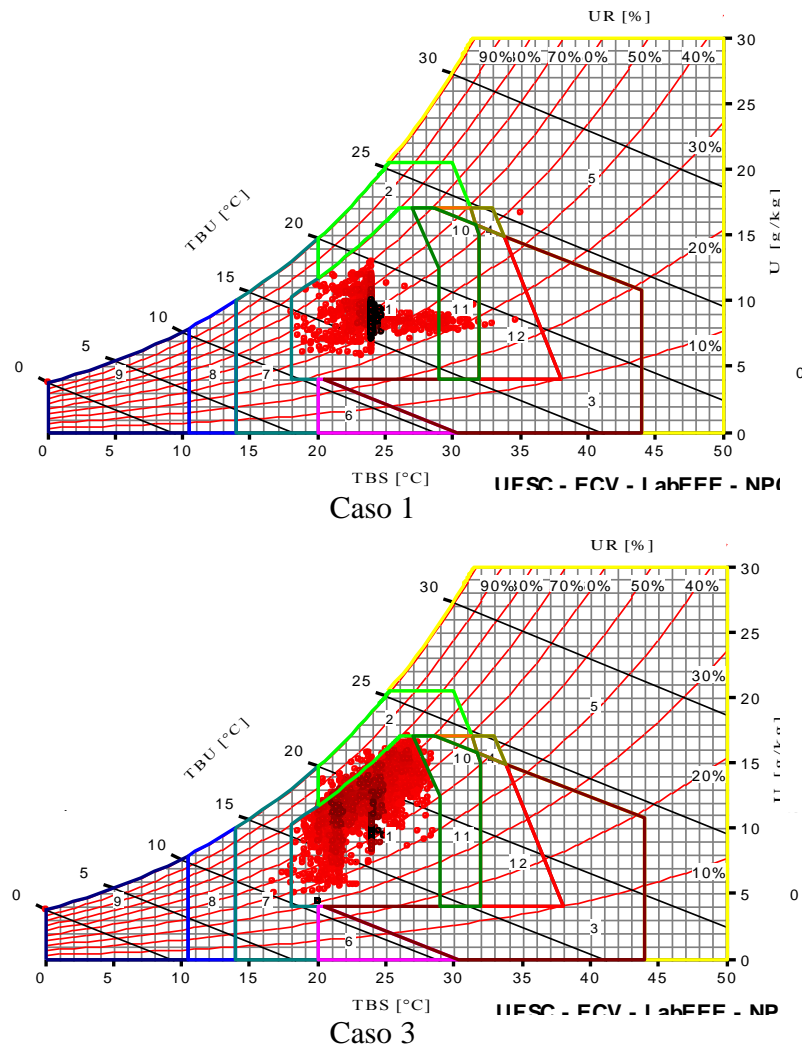

Caso 3

\section{Conclusões}

O potencial de economia de energia elétrica com a utilização da iluminação natural e da ventilação híbrida em edifícios comerciais localizados em Florianópolis foi estimado. Ressalta-se que não se considerou proteção à radiação solar direta. Assim, o potencial de economia de eletricidade para os casos com iluminação natural pode ter sido superestimado, porque, para evitar manchas de sol no interior dos modelos, o uso de elementos de proteção solar é recomendado. Se os elementos de proteção são externos, possivelmente o aproveitamento de luz natural é diminuído, mas também a carga térmica solar no interior do espaço é menor, acarretando redução do consumo do sistema de ar condicionado. Futuros trabalhos podem analisar a influência desses elementos de proteção no potencial de economia. Além disso, os potenciais de economia foram estimados tendo como parâmetro de conforto térmico o método de Givoni - o uso de outro método de conforto provavelmente conduziria a potenciais de economia diferentes. Outro fator importante a mencionar é que os modelos de ambientes de edifícios comerciais operaram com ventilação unilateral, o que pode reduzir significativamente o
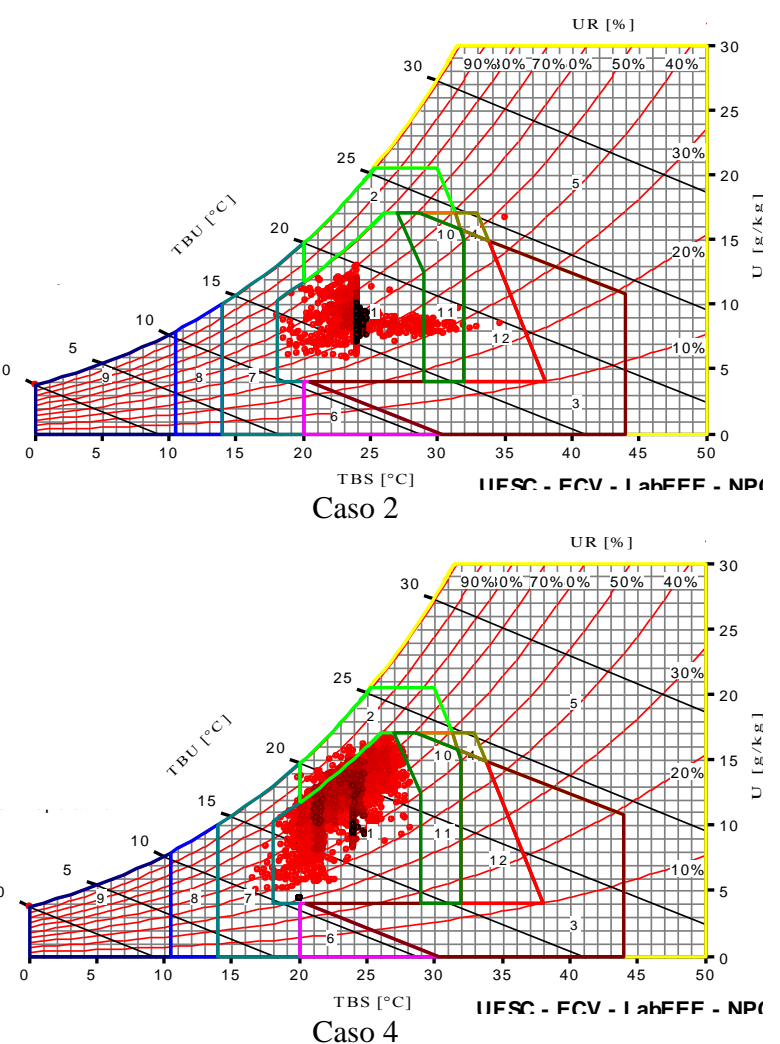

aproveitamento da ventilação natural. O uso de ventilação cruzada, por exemplo, influenciaria no potencial de economia (que poderia ser maior do que os valores apresentados neste artigo) para os casos com ventilação natural. Porém, o uso da ventilação natural deve ser estudado considerandose também a problemática do ruído trazido pela abertura das janelas. Em espaços com alto nível de ruído urbano, a abertura de janelas é restringida, o que afetaria os valores de potencial de economia apresentados neste trabalho. Desse modo, as conclusões deste trabalho são limitadas a essas considerações.

De maneira geral, pode-se concluir que a utilização da iluminação natural e da ventilação híbrida em edificações comerciais localizadas em Florianópolis apresenta potencial de economia de energia elétrica, quando comparado a edifícios com iluminação artificial e condicionamento artificial. Para ambientes operando com sistema de ar condicionado e utilizando iluminação natural integrada à iluminação artificial, o valor resultante de redução máxima no consumo total de energia elétrica foi de $50,5 \%$ em relação a edifícios com iluminação artificial e condicionamento artificial. Para ambientes operando com iluminação artificial e ventilação híbrida, chegou-se a uma redução de 
consumo total de energia elétrica de até $31,9 \%$ em relação a edifícios com iluminação artificial e condicionamento artificial. Quando se utiliza da iluminação natural e da ventilação natural, a economia de energia elétrica é ainda maior. Para ambientes operando com ventilação híbrida e com iluminação natural integrada ao sistema de iluminação artificial, a redução máxima no consumo total de energia elétrica foi de $64,9 \%$ em relação a edifícios com iluminação artificial e condicionamento artificial. Desse modo, ressaltase a importância em utilizarem-se a iluminação natural e a ventilação natural em edificações comerciais. Essas estratégias podem ser utilizadas para aumentar a eficiência energética em edifícios.

Nos ambientes condicionados artificialmente (Casos 1 e 2) há maior controle da umidade (a distribuição dos pontos na carta de Givoni é mais uniforme), quando comparada à dos ambientes com ventilação híbrida. Com a utilização da ventilação híbrida (Casos 3 e 4) há maior variação das propriedades do ar (maior dispersão dos pontos na zona de conforto térmico da carta de Givoni) com relação aos Casos 1 e 2. Isso ocorre porque se alterna entre o acionamento do sistema de ar condicionado e a ventilação natural durante o período de ocupação do edifício. Porém, mesmo assim, foi garantida uma elevada ocorrência de número de horas de conforto térmico para os ocupantes da edificação de segunda a sexta-feira das $8 \mathrm{~h}$ às $18 \mathrm{~h}$ (desconforto por calor pode ocorrer nos horários de início de operação do sistema de ar condicionado, e eventuais desconfortos por frio podem ocorrer no inverno, mesmo com as janelas fechadas).

\section{Referências}

\section{AMERICAN SOCIETY OF HEATING, REFRIGERATING AND AIR-CONDITIONING ENGINEERS. Standard 55: thermal environmental conditions for human occupancy. Atlanta, 2010.}

\section{ASSOCIAÇÃO BRASILEIRA DE NORMAS} TÉCNICAS. NBR ISO/CIE 8995-1: oluminação de ambientes de trabalho: parte 1: interior. Rio de Janeiro, 2013. 46 p.

BALANÇO ENERGÉTICO NACIONAL. Relatório final 2012: ano-base 2011. Rio de Janeiro: EPE, 2012. 282 p.

BODART, M.; HERDE, A. D. Global Energy Savings in Offices Buildings by the Use of Daylighting. Energy and Buildings, v. 34, n. 5, p. 421-429, 2002.
BRAGER, G.; BAKER, L. Occupant Satisfaction in Mixed-Mode Buildings. In: CONFERENCE AIR CONDITIONING AND THE LOW CARBON COOLING CHALLENGE, Cumberland Lodge, 2008. Proceedings... Cumberland Lodge, 2008. p. 27-29.

BRAGER, G.; BORGESON, S.; LEE, Y. S. Control Strategies For Mixed-Mode Buildings. Berkeley: Center for the Built Environment, University of California, 2007.

DAYSIM. Programa Computacional Daysim. Versão 3.0. Disponível em:

<http://www.daysim.com>. Acesso em: 15 maio 2011.

DIDONÉ, E. L; PEREIRA, F. O. R. Simulação Computacional Integrada Para a Consideração da Luz Natural na Avaliação do Desempenho Energético de Edificações. Ambiente Construído, v. 10 , n. 4, p. 139-154, out./dez. 2010.

EMMERICH, S. J.; POLIDORO, B.; AXLEY, J. W. Impact of Adaptive Thermal Comfort on Climatic Suitability of Natural Ventilation in Office Buildings. Energy and Buildings, v. 43, n. 9, p. 2101-2107, 2011.

ENERGY INFORMATION ADMINISTRATION. 2003 Commercial Buildings Energy Consumption Survey. U.S. Department of Energy. 2008.

ENERGYPLUS. DataSets. Lawrence Berkeley National Laboratory. V.6.0, 2010.

GIVONI, B. Comfort, Climate Analysis and Building Design Guidelines. Energy and Buildings, v. 18, n. 1, p. 11-23, 1992.

JI, Y.; LOMAS, K.J.; COOK, M.J. Hybrid Ventilation For Low Energy Building Design in South China. Building and Environment, v. 44, n. 11, p. 2245-2255, 2009.

KARAVA, P. et al. Experimental Study of the Thermal Performance of a Large Institutional Building With Mixed-Mode Cooling and Hybrid Ventilation. Building and Environment, v. 57, p. 313-326, 2012.

KRARTI, M.; ERICKSON, P.M.; HILLMAN, T.C. A Simplified Method to Estimate Energy Savings of Artificial Lighting Use From Daylighting. Building and Environment, v. 40, n. 6, p. 747-754, 2005.

LABORATÓRIO DE EFICIÊNCIA ENERGÉTICA EM EDIFICAÇÕES. Universidade Federal de Santa Catarina. Arquivo Climático de Florianópolis. Disponível em: <http://www.labeee.ufsc.br/>. Acesso em: $21 \mathrm{fev}$. 2011a. 


\section{LABORATÓRIO DE EFICIÊNCIA}

ENERGÉTICA EM EDIFICAÇÕES.

Universidade Federal de Santa Catarina.

Programa Computacional Analysis Bio. Versão

2.2. Disponível em:

$<$ http://www.labeee.ufsc.br/downloads/softwares/a nalysis-bio>. Acesso em: 15 maio 2011b.

LOMAS, K. J.; COOK, M. J.; FIALA, D. Low Energy Architecture For a Severe US Climate: design and evaluation of a hybrid ventilation strategy. Energy and Buildings, v. 39, n. 1, p. 3244, 2007.

LOUTZENHISER, P. G.; MAXWELL, G. M.; MANZ, H. An Empirical Validation of the Daylighting Algorithms and Associated Interactions in Building Energy Simulation Programs Using Various Shading Devices and Windows. Energy, v. 32, n. 10, p. 1855-1870, 2007.

MANKIBI, M. E. et al. Prediction of Hybrid Ventilation Performance Using Two Simulation Tools. Solar Energy, v. 80, n. 8, p. 908-926, 2006.

NIACHOU, K. et al. Experimental Performance Investigation of Natural, Mechanical and Hybrid Ventilation in Urban Environment. Building and Environment, v. 43, n. 8, p. 1373-1382, 2008.

PROGRAMA NACIONAL DE CONSERVAÇÃO DE ENERGIA ELÉTRICA. Avaliação do Mercado de Eficiência Energética no Brasil. 2007. Disponível em:

<www.eletrobras.gov.br/procel>. Acesso em: 2 fev. 2011.

RAMOS, G.; GHISI, E. Analysis of Daylight Calculated Using the EnergyPlus Program.

Renewable and Sustainable Energy Reviews, v. 14, n. 7, p. 1948-1958, 2010.
ROISIN, B. et al. Lighting Energy Savings in Offices Using Different Control Systems and Their Real Consumption. Energy and Buildings, v. 40, n. 4, p. 514-523, 2008.

RUPP, R. F. Dimensionamento de Área de Janela em Edificações Comerciais: integração da iluminação natural com a artificial e utilização da ventilação híbrida. Florianópolis, 2011.

Dissertação (Mestrado em Engenharia Civil) Faculdade de Engenharia Civil, Universidade Federal de Santa Catarina, Florianópolis, 2011.

RUPP, R. F.; GHISI, E. What Is the Most Adequate Method to Assess Thermal Comfort in Hybrid Commercial Buildings Located in HotHumid Summer Climate? Renewable and Sustainable Energy Reviews, v. 29, p. 449-462, jan. 2014.

SANTANA, M.V. Influência de Parâmetros Construtivos no Consumo de Energia de Edifícios de Escritório Localizados em Florianópolis-SC. Florianópolis, 2006. Dissertação (Mestrado em Engenharia Civil) Faculdade de Engenharia Civil, Universidade Federal de Santa Catarina, Florianópolis, 2006.

SORGATO, M. J. Desempenho Térmico de Edificações Residenciais Unifamiliares Ventiladas Naturalmente. Florianópolis, 2009. Dissertação (Mestrado em Engenharia Civil) Faculdade de Engenharia Civil, Universidade Federal de Santa Catarina, Florianópolis, 2009.

\section{SOUZA, M. B. Potencialidade de} Aproveitamento da Luz Natural Através da Utilização de Sistemas Automáticos de Controle Para Economia de Energia Elétrica.

Florianópolis, 2003. Tese (Doutorado em Engenharia de Produção) - Curso de PósGraduação em Engenharia de Produção, Universidade Federal de Santa Catarina, Florianópolis, 2003.

Ricardo Forgiarini Rupp

Departamento de Engenharia Civil, Centro Tecnológico | Universidade Federal de Santa Catarina | Rua Roberto Sampaio Gonzaga, s/n, Trindade | Florianópolis - SC - Brasil | CEP 88040-970 | Tel.: (48) 3721-9370 | E-mail: ricardorupp@gmail.com

Enedir Ghisi

Departamento de Engenharia Civil, Centro Tecnológico | Universidade Federal de Santa Catarina | Tel.: (48) 3721-2115 |

E-mail: enedir@labeee.ufsc.br

Revista Ambiente Construído

Associação Nacional de Tecnologia do Ambiente Construído

Av. Osvaldo Aranha, $99-3^{\circ}$ andar, Centro Porto Alegre - RS - Brasil

CEP 90035-190

Telefone: +55 (51) 3308-4084

Fax: +55 (51) 3308-4054

www.seer.ufrgs.br/ambienteconstruido

E-mail: ambienteconstruido@ufrgs.br

86 Rupp, R. F.; Ghisi, E. 